\title{
Numerical Approximations to Nonlinear Conservation Laws With Locally Varying Time and Space Grids
}

\author{
By Stanley Osher* and Richard Sanders**
}

\begin{abstract}
An explicit time differencing technique is introduced to approximate nonlinear conservation laws. This differencing technique links together an arbitrary number of space regimes containing fine and coarse time increments. Previous stability requirements, i.e. the CFL condition, placed a global bound on the size of the time increments. For scalar, monotone, approximations in one space dimension, using this variable step time differencing, convergence to the correct physical solution is proven given only a local CFL condition.
\end{abstract}

1. Introduction. We shall consider numerical approximations to the initial value problem for nonlinear systems of conservation laws

$$
\frac{\partial w}{\partial t}+\sum_{i=1}^{d} \frac{\partial}{\partial x_{i}} f_{i}(x, t, w)=g(x, t, w), \quad w(x, 0)=w_{0}(x) .
$$

Here $x=\left(x^{(1)}, \ldots, x^{(d)}\right) \in \mathbf{R}^{d}, w(x, t)$ is an $m$-vector of unknowns and each flux function, $f_{i}(x, t, w)$, is vector-valued having $m$ components. The system (1.1) is said to be hyperbolic when all eigenvalues of every real linear combination of the Jacobian matrices are real. It is well known that solutions of (1.1) may develop discontinuities in finite time, even when the initial data are smooth.

Among the numerical methods used to approximate discontinuous solutions of (1.1), those based on shock capturing have proved most successful. However, convergence of any explicit method can be possible only under a restrictive CFL condition. Another possibility is to use one of a variety of unconditionally stable implicit methods. One soon discovers that, in general, a nonlinear inversion must be implemented at each time step. Aside from the inherent computational complexity introduced by implementing such inversions, these techniques often fail to perform well for large time steps when nonsteady discontinuities are present.

For these reasons we shall consider explicit finite difference methods which use locally varying time grids. The global CFL restriction is replaced by a local restriction. Our goal is to develop a differencing technique at interface points between regions of distinct time increments. To do this we study the numerical flux function from a finite volume viewpoint. Here we stress that the finite volume construction yields an algorithm which is in conservation form, and in the scalar case satisfies a

Received September 14, 1982.

1980 Mathematics Subject Classification. Primary 65M10; Secondary 65M05.

${ }^{*}$ Research supported by NSF Grant \# MCS 82-007788 and NASA University Consortium Agreement \# NCA2-0R390-202.

**Research supported by NSF Grant \#MCS 82-00676. 
discrete version of the entropy inequality when applied to monotone numerical fluxes. Hence, no nonphysical limit solutions appear.

In Section 2 we will introduce a simplified version of our algorithm. We will adapt a standard explicit three-point conservation form difference scheme to a one-dimensional mesh containing two distinct time increments. This will motivate the more general version of our technique which will be discussed in Section 3. In Section 4 we will state and rigorously prove a convergence theorem (Theorem 3), for the scalar one-dimensional problem.

2. Preliminary Motivation. We begin our discussion by considering a simple yet illuminating example. Consider the one space dimensional scalar Cauchy problem:

$$
\frac{\partial w}{\partial t}+\frac{\partial}{\partial x} f(w)=0, \quad w(x, 0)=w_{0}(x)
$$

Many commonly used discrete approximations to this problem are obtained by a three-point conservation form difference scheme. Schemes of this type may be written as

$$
u_{j}^{n+1}=u_{j}^{n}-\frac{\Delta t}{\Delta x} \Delta_{+} h_{f}\left(u_{j}^{n}, u_{j-1}^{n}\right),
$$

where $u_{j}^{n}$ approximates $w(j \Delta x, n \Delta t), h_{f}$ is one of various numerical flux functions and $\Delta_{+}$denotes the forward difference operator. We now consider a mesh which contains two time increments, $\Delta t$ and $\Delta t / 2$. For this example we shall use $\Delta t$ for $j \leqslant j_{0}$ and $\Delta t / 2$ for $j \geqslant j_{0}+1$, where $j_{0}$ is some arbitrary integer.

Given $u_{j}^{n}$, we may obtain $u_{j}^{n+1}$ for $j \leqslant j_{0}-1$ via the difference equation (2.2). For $j \geqslant j_{0}+2$ one can replace $\Delta t$ in (2.2) with $\Delta t / 2$. This gives the difference equation

$$
u_{j}^{n+1 / 2}=u_{j}^{n}-\frac{1}{2} \frac{\Delta t}{\Delta x} \Delta_{+} h_{f}\left(u_{j}^{n}, u_{j-1}^{n}\right) .
$$

For these values of $j, u_{j}^{n+1}$ can be obtained from $u_{j}^{n}$ by composing (2.3) with itself. Schematically, the resulting difference stencil is:

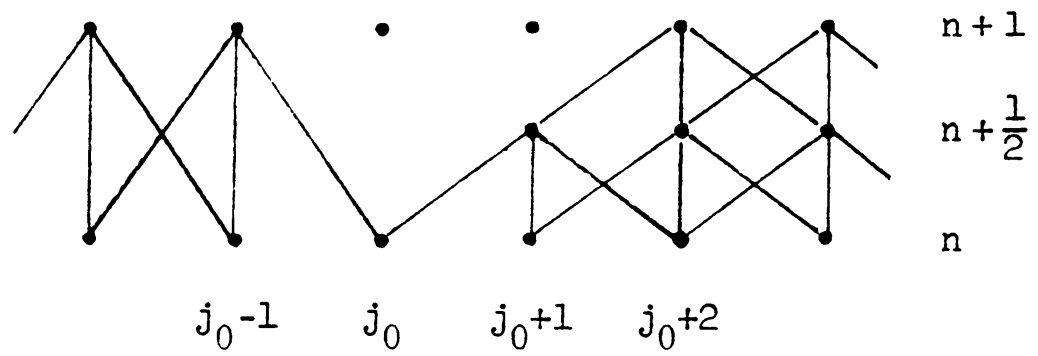

FIGURE 1

The only quantities whose evaluations require some thought are $u_{j_{0}}^{n+1}$ and $u_{j_{0}+1}^{n+1}$, the so-called interface values.

By way of motivation we briefly return to a mesh with constant time increments. The grid functions, $u_{j}^{n}$, may be regarded as the values of a step function, $u^{\Delta}(x, t)$, defined by

$$
u^{\Delta}(x, t)=u_{j}^{n}
$$


when $x \in[j \Delta x,(j+1) \Delta x)$ and $t \in[n \Delta t,(n+1) \Delta t)$. We now make a standard, but important observation. The difference equation (2.2) may be written in terms of the step function (2.4). We write

$$
\begin{aligned}
\int_{j \Delta x}^{(j+1) \Delta x}\left[u^{\Delta}(x,(n+1) \Delta t)-u^{\Delta}(x, n \Delta t)\right] d x \\
+\int_{n \Delta t}^{(n+1) \Delta t}\left[h_{f}\left(u^{\Delta}((j+1) \Delta x, t), u^{\Delta}(j \Delta x, t)\right)\right. \\
\left.-h_{f}\left(u^{\Delta}(j \Delta x, t), u^{\Delta}((j-1) \Delta x, t)\right)\right] d t=0 .
\end{aligned}
$$

The formulation (2.5) now allows us to derive a scheme to combine (2.2) with (2.3). For $x<\left(j_{0}+1\right) \Delta x$ we define the step function $u^{\Delta}(x, t)$ to be

$$
u^{\Delta}(x, t)=u_{j}^{n}
$$

when $x \in[j \Delta x,(j+1) \Delta x)$ and $t \in[n \Delta t,(n+1) \Delta t)$. For $x \geqslant\left(j_{0}+1\right) \Delta x$ we define $u^{\Delta}(x, t)$ to be

$$
u^{\Delta}(x, t)=u_{j}^{n},
$$

when $x \in[j \Delta x,(j+1) \Delta x)$ and $t \in\left[n \Delta t,\left(n+\frac{1}{2}\right) \Delta t\right)$ and

$$
u^{\Delta}(x, t)=u_{j}^{n+1 / 2} \text {, }
$$

when $x \in[j \Delta x,(j+1) \Delta x)$ and $t \in\left[\left(n+\frac{1}{2}\right) \Delta t,(n+1) \Delta t\right)$. If, for $j \leqslant j_{0}-1$, we insert (2.6) into (2.5), the difference equation (2.2) is obviously recovered. On the other hand, for $j \geqslant j_{0}+2$, inserting (2.6) and (2.7) into (2.5) yields the composite of Eq. (2.3). At the interface, we find that (2.5) applied to the step function defined by (2.6), (2.7) and (2.8) gives us the interface difference equations

$$
u_{j_{0}}^{n+1}=u_{j_{0}}^{n}-\frac{\Delta t}{\Delta x}\left[\frac{1}{2}\left(h_{f}\left(u_{j_{0}+1}^{n+1 / 2}, u_{j_{0}}^{n}\right)+h_{f}\left(u_{j_{0}+1}^{n}, u_{j_{0}}^{n}\right)\right)-h_{f}\left(u_{j_{0}}^{n}, u_{j_{0}-1}^{n}\right)\right]
$$

and

$$
u_{j_{0}+1}^{n+1}=u_{j_{0}+1}^{n+1 / 2}-\frac{1}{2} \frac{\Delta t}{\Delta x}\left[h_{f}\left(u_{j_{0}+2}^{n+1 / 2}, u_{j_{0}+1}^{n+1 / 2}\right)-h_{f}\left(u_{j_{0}+1}^{n+1 / 2}, u_{j_{0}}^{n}\right)\right]
$$

Difference equations (2.2), (2.3), (2.9) and (2.10) may be written in a two-step, predictor-corrector type form. We write the predictor as

$$
u_{j}^{n+1 / 2}=\left\{\begin{array}{l}
u_{j}^{n}, \quad j \leqslant j_{0}, \\
u_{j}^{n}-\frac{1}{2} \frac{\Delta t}{\Delta x} \Delta_{+} h_{f}\left(u_{j}^{n}, u_{j-1}^{n}\right), \quad j \geqslant j_{0}+1,
\end{array}\right.
$$

and the corrector as

$$
u_{j}^{n+1}=u_{j}^{n}-\frac{1}{2} \frac{\Delta t}{\Delta x} \Delta_{+}\left[h_{f}\left(u_{j}^{n}, u_{j-1}^{n}\right)+h_{f}\left(u_{j}^{n+1 / 2}, u_{j-1}^{n+1 / 2}\right)\right] .
$$

This example motivates the construction for the general problem (1.1) which is contained in Section 3.

The simple one-step predictor will, in general, be replaced by an $M-1$ step predictor. We shall allow an arbitrary number of local refinements in time when advancing between time steps $t^{n}$ and $t^{n+1}$. 
3. The Finite Volume Method. A notion fundamental to our approach is that of the numerical flux. We exemplify this by considering the one space dimensional problem:

$$
\frac{\partial w}{\partial t}+\frac{\partial f}{\partial x}(x, t, w)=g(x, t, w)
$$

We partition the real line into intervals

$$
q_{j}=\left\{x: x_{j-1 / 2} \leqslant x<x_{j+1 / 2}\right\},
$$

with $\Delta x_{j}=x_{j+1 / 2}-x_{j-1 / 2}$ and $x_{j}$ the midpoint of $\mathscr{G}_{j}$. Assume that $w\left(x, t_{0}\right)$ is given. We then integrate (3.1) over $\mathscr{G}_{j}$, divide by $\Delta x_{j}$ and arrive at

$$
\begin{aligned}
& \left.\frac{\partial}{\partial t} \frac{1}{\Delta x_{j}} \int_{G_{j}} w(x, t) d x\right|_{t=t_{0}} \\
& \quad=-\frac{1}{\Delta x_{j}} \Delta_{+} f\left(x_{j-1 / 2}, t_{0}, w\left(x_{j-1 / 2}, t_{0}\right)\right)+\frac{1}{\Delta x_{j}} \int_{G_{j}} g\left(x, t_{0}, w\left(x, t_{0}\right)\right) d x .
\end{aligned}
$$

Here, we recall the forward difference operator is defined to be

$$
\Delta_{+} f_{j}=f_{j+1}-f_{j}
$$

and we shall use $\Delta_{+}^{t}$ or $\Delta_{+}^{x}$ with superscripts when necessary.

Define $\chi_{g},(x)$ to be the characteristic function of $\oint_{j}$, that is:

$$
\chi_{g_{j}}(x)= \begin{cases}1, & x \in g_{j}, \\ 0, & x \notin g_{j} .\end{cases}
$$

Let $u^{\Delta}(x, t)$ be a semidiscrete approximation to $w$ defined by

$$
u^{\Delta}(x, t)=\sum_{j} u_{j}^{\Delta}(t) \chi_{q_{1}}(x)
$$

The superscript $\Delta$ is equal to $\max _{j} \Delta x_{j}$ and will denote the measure of grid refinement. We then replace $f$ in (3.2) by a numerical flux function $h_{f}$, and let $u_{j}^{\Delta}(t)$, which is to approximate $\left(1 / \Delta x_{j}\right) \int_{g_{j}} w(x, t) d x$, evolve via the system of ordinary differential equations:

$$
\begin{aligned}
\frac{\partial u_{j}^{\Delta}(t)}{\partial t}= & -\frac{1}{\Delta x_{j}} \Delta_{+} h_{f}\left(x_{j-1 / 2}, t, u_{j}^{\Delta}(t), u_{j-1}^{\Delta}(t)\right) \\
& +\frac{1}{\Delta x_{j}} \int_{g_{j}} g\left(x, t, u_{j}^{\Delta}(t)\right) d x .
\end{aligned}
$$

The numerical flux function, $h_{f}$, is defined for every smooth $m$-vector $f$. It is furthermore assumed to be a Lipschitz continuous function of $x, t, u_{j}$ and $u_{j-1}$ subject to the consistency requirement

$$
h_{f}(x, t, u, u)=f(x, t, u) .
$$

Examples of such numerical fluxes will be given below. We note that the dependence of $h_{f}$ is only on the two values of $u^{\Delta}$ adjacent to the boundary. This may restrict the accuracy of the approximation. Nevertheless, several of these approximations perform exceptionally well for flows having strong shocks, provided that the flow is close to steady state [1], [7], [16]. 
Next we consider the general multi-dimensional problem (1.1). We decompose $\mathbf{R}^{d}$ into nonoverlapping polyhedra

$$
\mathbf{R}^{d}=\bigcup_{j} \Omega_{j}^{\Delta}
$$

for $\Delta$ a measure of refinement to be defined below and $\Omega_{j}^{\Delta}$ a polyhedron.

We assume the following property: If $\bar{\rho}\left(\Omega_{j}^{\Delta}\right)$ (resp. $\left.\rho\left(\Omega_{j}^{\Delta}\right)\right)$ is the smallest (largest) diameter of the ball containing (contained in) $\Omega_{j}^{\Delta}$, there exists a positive constant $K_{1}$ such that

$$
K_{1}^{-1} \Delta \leqslant \inf _{j} \underline{\rho}\left(\Omega_{j}^{\Delta}\right) \leqslant \sup _{j} \bar{\rho}\left(\Omega_{j}^{\Delta}\right) \leqslant K_{1} \Delta .
$$

We also define $\left|\Omega_{j}^{\Delta}\right|$ to be the volume of each $\Omega_{j}^{\Delta}$.

The analogue of (3.2) for the multi-dimensional problem is

$$
\begin{aligned}
& \left.\frac{\partial}{\partial t} \frac{1}{\left|\Omega_{j}^{\Delta}\right|} \int_{\Omega_{j}^{\Delta}} w(x, t) d x\right|_{t=t_{0}} \\
& \quad=-\frac{1}{\left|\Omega_{j}^{\Delta}\right|} \int_{\partial \Omega_{j}^{\Delta}}(F \cdot n)\left(x, t_{0}, w\left(x, t_{0}\right)\right) d s+\frac{1}{\left|\Omega_{j}^{\Delta}\right|} \int_{\Omega_{j}^{\lrcorner}} g\left(x, t_{0}, w\left(x, t_{0}\right)\right) d x .
\end{aligned}
$$

Here $F$ is the $m \times d$ matrix

$$
F=\left(f_{1}, \ldots, f_{d}\right)
$$

and $n=\left(n_{x^{(1)}}, \ldots, n_{x^{(d)}}\right)$ is the piecewise constant outward normal to $\Omega_{j}^{\Delta}$. Thus, for each boundary face the vector function

$$
F \cdot n=\sum_{i=1}^{d} n_{x^{(1)}} f_{i}
$$

is a one space dimensional flux function. For these functions, we have already defined a class of numerical flux functions, which we now write as

$$
h_{F \cdot n}\left(x, t, u_{1}, u_{2}\right)
$$

with consistency implying that

$$
h_{F \cdot n}(x, t, u, u)=(F \cdot n)(x, t, u),
$$

and conservation form given by

$$
h_{F \cdot(-n)}\left(u_{1}, u_{2}\right)=-h_{F \cdot n}\left(u_{2}, u_{1}\right) .
$$

The surface integral in (3.8) is approximated in the following manner. On each planar segment of boundary, $P_{j, l}^{\Delta}$, we approximate

$$
\int_{P_{j, l}^{\Delta}}\left(F \cdot n_{j, l}^{\Delta}\right)\left(x, t_{0}, w\left(x, t_{0}\right)\right) d s
$$

by

$$
\int_{P_{j, l}^{\Delta}} h_{F \cdot n_{j, l}^{\Delta}}\left(x, t_{0}, \bar{u}_{j, l}^{\Delta}, \underline{u}_{j . l}^{\Delta}\right) d s .
$$

Here, $\bar{u}_{j, l}^{\Delta}$ is the outer trace of $u^{\Delta}\left(x, t_{0}\right)$ on $P_{j, l}^{\Delta}$, and $\underline{u}_{j, l}^{\Delta}$ is the inner trace of $u^{\Delta}\left(x, t_{0}\right)$ on $P_{j, l}^{\Delta}$. 
In this manner we may start from any one space dimensional numerical flux function and create a multi-dimensional finite volume algorithm. This is done by obtaining $h_{F \cdot n}$ at all boundaries.

In the case when $F \cdot n$ does not depend explicitly on the tangential component of $x$, the right-hand side of $(3.11)$ is trivially computed since the integrand is piecewise constant.

The semidiscrete finite volume approximation to (1.1) is obtained by writing

$$
u^{\Delta}(x, t)=\sum_{j} u_{j}^{\Delta}(t) \chi_{\Omega_{j}^{\Delta}(x)}
$$

and allowing $u_{j}^{\Delta}(t)$ to evolve via the system of ordinary differential equations:

$$
\frac{\partial}{\partial t} u_{j}^{\Delta}=-\frac{1}{\left|\Omega_{j}^{\Delta}\right|} \int_{\partial \Omega_{j}^{\Delta}} h_{F \cdot n}\left(x, t, \bar{u}_{j}^{\Delta}, \underline{u}_{j}^{\Delta}\right) d s+\frac{1}{\left|\Omega_{j}^{\Delta}\right|} \int_{\Omega_{j}^{\Delta}} g\left(x, t, u_{j}^{\Delta}\right) d x .
$$

In the scalar case it is not difficult to justify this construction when $h_{f}$ corresponds to a monotone flux function. The resulting numerical flux function (3.10) is said to be monotone if it is both nonincreasing in $u_{1}$ and nondecreasing in $u_{2}$.

We pause for a moment to present three examples of such numerical flux functions.

(A) The Godunov scheme [6]. Here the true solution to the Riemann initial value problem is computed and evaluated at $x=x_{j-1 / 2}, t \geqslant t_{0}$ for $t-t_{0}$ small. The Riemann initial data is $w\left(x, t_{0}\right) \equiv u_{j-1}$ for $x \leqslant x_{j-1 / 2}$ and $w\left(x, t_{0}\right) \equiv u_{j}$ for $x>$ $x_{j-1 / 2}$. The true solution satisfies the entropy condition (E) [9], [10], [13]. In the present case, this procedure yields a similarity solution which is constant along rays $\left(x-x_{j-1 / 2}\right) /\left(t-t_{0}\right)=$ constant. Thus the numerical flux function is defined by

$$
h_{f}\left(u_{j}, u_{j-1}\right)=f\left(w\left(x_{j-1 / 2}, t_{0}^{+}\right)\right)=\lim _{t \downarrow t_{0}} f\left(w\left(x_{j-1 / 2}, t\right)\right) .
$$

For nonconvex $f$ this algorithm can become fairly complicated. Furthermore the numerical flux function does not have continuous first partial derivatives with respect to its arguments. The derivatives are discontinuous when $u_{j}$ and $u_{j-1}$ are connected by a single steady shock [5]. In general this scheme does resolve steady shocks with a one-point transition.

(B) The Engquist-Osher sheme [3], [4]. Let the increasing and decreasing parts of $f(u)$ be computed exactly by

$$
f_{+}(u)=\int_{0}^{u} \max \left(f^{\prime}(s), 0\right) d s, \quad f_{-}(u)=\int_{0}^{u} \min \left(f^{\prime}(s), 0\right) d s,
$$

where $f$ is normalized so that $f(0)=0$. Then we define

$$
h_{f}\left(u_{j}, u_{j-1}\right)=f_{-}\left(u_{j}\right)+f_{+}\left(u_{j-1}\right) .
$$

This flux function will in general be less complicated than Godunov's. It will have continuous first partial derivatives and will in general resolve steady shocks with a two-point transition. These two properties are related, see [5].

(C) The Lax-Friedrichs scheme [10]. Although this scheme was originally derived as an explicit time marching algorithm, we can construct a semidiscrete analogue with

$$
h_{f}\left(u_{j}, u_{j-1}\right)=\frac{1}{2}\left(f\left(u_{j}\right)+f\left(u_{j-1}\right)\right)-\frac{K}{2}\left(u_{j}-u_{j-1}\right)
$$

for some positive constant $K$ such that $\left|f^{\prime}(u)\right| \leqslant K$. 
This numerical flux function is the simplest to compute and is smooth. However, the resulting algorithm smears discrete shocks excessively, [8], [4].

We can now state two theorems and a remark concerning semidiscrete monotone finite volume approximations for the scalar and homogeneous version of (1.1). The proofs of Theorem 1 and Theorem 2 below were given in [14] for the Engquist-Osher scheme. However, there is no difficulty extending these results to any monotone scheme.

THEOREM 1. Let $u^{\Delta}(x, t)$ and $v^{\Delta}(x, t)$ be defined by (3.12) and the scalar, homogeneous version of (3.13). If the numerical flux function in (3.13) is monotone, we have for any $t_{1} \geqslant t_{0} \geqslant 0$

$$
\int_{\mathbf{R}^{d}}\left|u^{\Delta}\left(x, t_{1}\right)-v^{\Delta}\left(x, t_{1}\right)\right| d x \leqslant \int_{\mathbf{R}^{d}}\left|u^{\Delta}\left(x, t_{0}\right)-v^{\Delta}\left(x, t_{0}\right)\right| d x .
$$

Definition. For a scalar function of the type (3.12) we define its space variation as

$$
\operatorname{Var}\left(u^{\Delta}\right)=\sum_{j, l}\left|u^{\Delta}\left(x_{j}\right)-u^{\Delta}\left(x_{l}\right)\right| \Delta^{d-1},
$$

where each $x_{j}$ is the centroid of $\Omega_{j}^{\Delta}$. The sum is taken over all $j, l$ such that $\Omega_{j}^{\Delta}$ and $\Omega_{l}^{\Delta}$ have a common planar boundary.

THEOREM 2. Suppose that $u^{\Delta}(x, t)$ is defined as in Theorem 1. Furthermore, suppose that the $\operatorname{Var}\left(u^{\Delta}(\cdot, t)\right)$ remains uniformly bounded for all $\Delta$ tending to zero and all $t \in[0, T]$. Then the $\lim _{\Delta \downarrow 0} u^{\Delta}(x, t)$ exists in $L_{\text {loc }}^{1}\left(\mathbf{R}^{d}\right)$ on the bounded strip $t \in[0, T]$, and the limit is the unique entropy condition satisfying weak solution of the scalar and homogeneous version of Eq. (1.1).

Remark 1. If $\left\{\Omega^{\Delta}\right\}$ is constructed using the tensor product of one-dimensional (possibly variable) spatial grids and if the initial data are in $L^{\infty} \cap L^{1} \cap B V$, then the assumptions of Theorem 2 are valid. See [15].

We now turn to the time discretization of this method. A simple explicit method is obtained by defining $u_{j}^{\Delta}\left(t^{n}\right)$ in the usual fashion with $\Delta t^{n}=t^{n+1}-t^{n}$ and approximating the differential system

$$
\frac{\partial u_{j}^{\Delta}}{\partial t}=\left.\mathscr{H}\left(x_{j}, t, \bar{u}_{j}^{\Delta}, \underline{u}_{j}^{\Delta}\right)\right|_{t=t^{n}}
$$

by

$$
u_{j}^{\Delta}\left(t^{n+1}\right)=u_{j}^{\Delta}\left(t^{n}\right)+\Delta t^{n} \mathcal{H}\left(x_{j}, t^{n}, \bar{u}_{j}^{\Delta}\left(t^{n}\right), \underline{u}_{j}^{\Delta}\left(t^{n}\right)\right) .
$$

The drawback here is that the convergence of this method is possible only under a restrictive CFL condition. The analogues of Theorem 1, Theorem 2 and Remark 1 above can be proven [14], [15] under the restriction:

$$
\max _{j} \frac{\Delta t}{\left|\Omega_{j}^{\Delta}\right|} \int_{\partial \Omega_{j}^{\Delta}} \frac{\partial}{\partial \underline{u}}\left(h_{F \cdot n}\right)(x, t, \bar{u}, \underline{u}) d s \leqslant 1 .
$$

(For $F$ explicitly independent of $x, t$, the condition need only be checked for all $u$ contained in the convex hull of the initial data. For nondifferentiable but Lipschitz continuous flux functions, the derivative may be replaced by the Lipschitz constant.)

This global restriction, (3.20), is what we shall remove below by using local explicit time discretization. 
3.2. Local Time Discretization. We begin this section by considering the autonomous and homogeneous versions of (3.1).

Partition the space axis into a union of disjoint intervals

$$
\mathbf{R}=\bigcup_{j} q_{j}
$$

At each time level $t^{n}$, decompose this partition into two subsets $\cup_{j \in \mathfrak{e}^{n}} \Psi_{j}$ and $\cup_{j \notin e^{n}} q_{j}$, where $e^{n}$ is any subset of the integers (possibly dependent on $n$ ). The time increment $\left[t^{n}, t^{n+1}\right)$ is associated with those $j$ 's belonging to $e^{n}$. Otherwise, we partition $\left[t^{n}, t^{n+1}\right)$ into $\bigcup_{l=0}^{M-1}\left[t^{n+\eta_{l}}, t^{n+\eta_{l+1}}\right)$, where $t^{n+\eta_{l}}$ is defined below, and associate these time increments to those $j$ 's not belonging to $e^{n}$.

Let $\left\{\sigma_{k}\right\}_{k=1}^{M}$ be a sequence of positive numbers such that $\sum_{k=1}^{M} \sigma_{k}=1$. Define $\eta_{l}=\sigma_{1}+\cdots+\sigma_{l}$ with $\eta_{0} \equiv 0$. We now define $t^{n+\eta_{l+1}}=t^{n+\eta_{l}}+\sigma_{l+1} \Delta t^{n}$.

We propose to advance from time level $t^{n}$ to time level $t^{n+1}$ via a predictor-corrector type method.

The predictor is as follows:

For $k=1, \ldots, M-1$

$$
u_{j}\left(t^{n+\eta_{k}}\right)=\left\{\begin{array}{l}
u_{j}\left(t^{n}\right), \quad j \in e^{n}, \\
u_{j}\left(t^{n}\right)-\lambda_{j}^{n} \sum_{l=0}^{k-1} \sigma_{l+1} \Delta_{+} h_{f}\left(u_{j}\left(t^{n+\eta_{l}}\right), u_{j-1}\left(t^{n+\eta_{l}}\right)\right), \quad j \notin e^{n},
\end{array}\right.
$$

where $\lambda_{j}^{n}$ will be defined as $\Delta t^{n} / \Delta x_{j}$ and, from now on, the superscript $\Delta$ will be ignored. The corrector is:

$$
u_{j}\left(t^{n+1}\right)=u_{j}\left(t^{n}\right)-\lambda_{j}^{n} \sum_{l=0}^{M-1} \sigma_{l+1} \Delta_{+} h_{f}\left(u_{j}\left(t^{n+\eta_{l}}\right), u_{j-1}\left(t^{n+\eta_{l}}\right)\right) .
$$

This approach will later be justified.

We note that if $j-1, j$ and $j+1$ all belong to $e^{n}$ the algorithm reduces to

$$
u_{j}\left(t^{n+1}\right)=u_{j}\left(t^{n}\right)-\lambda_{j}^{n} \Delta_{+} h_{f}\left(u_{j}\left(t^{n}\right), u_{j-1}\left(t^{n}\right)\right) .
$$

Furthermore, for $j$ not belonging to $e^{n}$, the algorithm may be written inductively as

$$
u_{j}\left(t^{n+\eta_{k+1}}\right)=u_{j}\left(t^{n+\eta_{k}}\right)-\lambda_{j}^{n} \sigma_{k+1} \Delta_{+} h\left(u_{j}\left(t^{n+\eta_{k}}\right), u_{j-1}\left(t^{n+\eta_{k}}\right)\right)
$$

for $k=0, \ldots, M-1$. Thus, the necessary computer programming is quite simple. Values of $u_{j}$ at the same time level depend only on the values of $u_{j-1}, u_{j}$ and $u_{j+1}$ at the previous time level, except when $j$ belongs to $e^{n}$ and either $j-1$ or $j+1$ does not. We call such points $x_{j}$ interface points. For these points, we must store the associated neighboring values of $u_{j}$ at all $M-1$ intermediate time levels so that $u_{j}$ may be advanced from $t^{n}$ to $t^{n+1}$.

In Section 4 we shall prove convergence for the scalar and monotone versions of this algorithm subject to a local CFL condition. The remainder of the present section will be devoted to motivation and generalization of the previous algorithm (3.21), (3.22). 
Suppose that we integrate (3.8) over the time interval $\left[t^{\prime}, t^{\prime \prime}\right]$. We then obtain

$$
\begin{aligned}
& \frac{1}{\left|\Omega_{j}\right|} \int_{\Omega_{j}} w\left(x, t^{\prime \prime}\right) d x \\
& =\frac{1}{\left|\Omega_{j}\right|} \int_{\Omega_{j}} w\left(x, t^{\prime}\right) d x-\frac{1}{\left|\Omega_{j}\right|} \int_{t^{\prime}}^{t^{\prime \prime}} \int_{\partial \Omega_{j}}(F \cdot n)(x, t, w(x, t)) d s d t \\
& \quad+\frac{1}{\left|\Omega_{j}\right|} \int_{t^{\prime}}^{t^{\prime \prime}} \int_{\Omega_{j}} g(x, t, w(x, t)) d x d t .
\end{aligned}
$$

Next, we partition $\mathbf{R}^{d}$ as before, again decomposing this partition into two subsets $\cup_{j \in \mathbb{e}^{n}} \Omega_{j}$ and $\cup_{j \notin \mathbb{e}^{n}} \Omega_{j}$. For $j$ belonging to $e^{n}$ define

$$
u_{j}(t)=u_{j}\left(t^{n}\right), \quad \text { when } t \in\left[t^{n}, t^{n+1}\right),
$$

and for $j$ not belonging to $e^{n}$ define

$$
u_{j}(t)=u_{j}\left(t^{n+\eta_{k}}\right), \quad \text { when } t \in\left[t^{n+\eta_{h}}, t^{n+\eta_{k+1}}\right) .
$$

In (3.25) we let $t^{\prime}=t^{n}$ and $t^{\prime \prime}=t^{n+1}$ for $j \in \varrho^{n}$ or for $j \notin \bigodot^{n}$ we let $t^{\prime}=t^{n+\eta_{k}}$ and $t^{\prime \prime}=t^{n+\eta_{k+1}}, 0 \leqslant k \leqslant M-1$. Formally substituting $h_{F \cdot n}$ for $F \cdot n$ and inserting (3.26) and (3.27) into (3.25), we obtain a discrete finite volume approximation to (1.1): For $j \in e^{n}$ we have

$$
\begin{aligned}
u_{j}\left(t^{n+1}\right)= & u_{j}\left(t^{n}\right)-\frac{1}{\left|\Omega_{j}\right|} \int_{t^{n}}^{t^{n+1}} \int_{\partial \Omega} h_{F \cdot n}\left(x, t, \bar{u}_{j}(t), \underline{u}_{j}(t)\right) d s d t \\
& +\frac{1}{\left|\Omega_{j}\right|} \int_{t^{n}}^{t^{n+1}} \int_{\Omega_{j}} g\left(x, t, u_{j}\left(t^{n}\right)\right) d x d t .
\end{aligned}
$$

For $j \notin \mathcal{C}^{n}$ we have

$$
\begin{aligned}
u_{j}\left(t^{n+\eta_{k+1}}\right) & \\
= & u_{j}\left(t^{n+\eta_{k}}\right)-\frac{1}{\left|\Omega_{j}\right|} \int_{t^{n+\eta_{k}}}^{t^{n+\eta_{k+1}}} \int_{\partial \Omega_{j}} h_{F \cdot n}\left(x, t, \bar{u}_{j}\left(t^{n+\eta_{k}}\right), \underline{u}_{j}\left(t^{n+\eta_{k}}\right)\right) d s d t \\
& +\frac{1}{\left|\Omega_{j}\right|} \int_{t^{n+\eta_{k}}} \int_{\Omega_{j}} g\left(x, t, u_{j}\left(t^{n+\eta_{k}}\right)\right) d x d t
\end{aligned}
$$

where $k=0, \ldots, M-1$. This algorithm can be cast into predictor-corrector form. The predictor becomes:

For $k=1, \ldots, M-1$

$$
\begin{aligned}
& u_{j}\left(t^{n+\eta_{k}}\right) \\
& =\left\{\begin{aligned}
u_{j}\left(t^{n}\right), \quad j \in \mathbb{C}^{n}, \\
u_{j}\left(t^{n}\right)-\frac{1}{\left|\Omega_{j}\right|} \sum_{l=0}^{k-1}\left[\int_{t^{n+\eta_{l}}}^{t^{n+\eta_{l+1}}} \int_{\partial \Omega_{j}} h_{F \cdot n}\left(x, t, \bar{u}_{j}\left(t^{n+\eta_{l}}\right), \underline{u}_{j}\left(t^{n+\eta_{l}}\right)\right) d s d t\right. \\
\left.\quad+\int_{t^{n+\eta_{l}}}^{t^{n+\eta_{l+1}}} \int_{\Omega_{j}} g\left(x, t, u_{j}\left(t^{n+\eta_{l}}\right)\right) d x d t\right], j \notin \mathbb{C}^{n} .
\end{aligned}\right.
\end{aligned}
$$


The corrector becomes:

$$
\begin{aligned}
u_{j}\left(t^{n+1}\right)= & u_{j}\left(t^{n}\right) \\
& -\frac{1}{\left|\Omega_{j}\right|} \sum_{l=0}^{M-1}\left[\int_{t^{n+\eta_{l}}}^{t^{n+\eta_{l+1}}} \int_{\partial \Omega_{j}} h_{F \cdot n}\left(x, t, \bar{u}_{j}\left(t^{n+\eta_{l}}\right), \underline{u}_{j}\left(t^{n+\eta_{l}}\right)\right) d s d t\right. \\
& \left.\quad+\int_{t^{n+\eta_{l}}}^{t^{n+\eta_{l+1}}} \int_{\Omega_{j}} g\left(x, t, u_{j}\left(t^{n+\eta_{l}}\right)\right) d x d t\right] .
\end{aligned}
$$

For the one-dimensional case, when $F$ is explicitly independent of $x$ and $t$ and $g=0$, (3.30), (3.31) reduce to (3.22), (3.23).

4. Statement and Proof of Theorem 3. In this section we state and prove a convergence theorem for a particular case of algorithm (3.21), (3.22). We shall restrict our attention to the equation

$$
\frac{\partial w}{\partial t}+\frac{\partial}{\partial x} f(w)=0, \quad w(x, 0)=w_{0}(x)
$$

where $w(x, t)$ is a scalar, $f(w)$ is locally Lipschitz continuous and the initial data are in the space $L^{\infty} \cap L^{1} \cap B V$.

So that this section is self-contained, algorithm (3.21), (3.22) shall be restated for the particular case applied to (4.1).

Let the values of the step function, $u^{\Delta}(x, t)$, be defined as

$$
\begin{aligned}
& u^{\Delta}(x, t)=u_{j}^{n}, \quad \text { if } x \in\left[x_{j-1 / 2}, x_{j+1 / 2}\right), t \in\left[t^{n}, t^{n+1}\right) \text { and } j \in \mathcal{C}^{n}, \\
& \text { and } \\
& u^{\Delta}(x, t)=u_{j}^{n+\eta_{l}}, \quad \text { if } x \in\left[x_{j-1 / 2}, x_{j+1 / 2}\right), t \in\left[t^{n+\eta_{l}}, t^{n+\eta_{l+1}}\right) \text { and } j \notin \mathcal{C}^{n},
\end{aligned}
$$

where $e^{n}$ is any subset of the integers (possibly dependent on $n$ ); $\eta_{l}=\sigma_{1}+\cdots+\sigma_{l}$ with $\eta_{0} \equiv 0$ where $\left\{\sigma_{k}\right\}_{k=1}^{M}$ is any sequence of positive numbers such that $\sum_{k=1}^{M} \sigma_{k}=1$; and $t^{n+\eta_{l}}$ is given by $t^{n+\eta_{l+1}}=t^{n+\eta_{l}}+\sigma_{l+1} \Delta t^{n}, l=0, \ldots, M-1$. The superscript $\Delta$ on $u^{\Delta}(x, t)$ denotes a measure of grid refinement, $\Delta=\max _{j, n}\left(\Delta x_{j}, \Delta t^{n}\right)$.

The values $u_{j}^{n+\eta_{k}}, k=1, \ldots, M-1$, are obtained from $u_{j}^{n}$ via the predictor

$$
u_{j}^{n+\eta_{k}}=\left\{\begin{array}{l}
u_{j}^{n}, \quad j \in \bigodot^{n}, \\
u_{j}^{n}-\lambda_{j}^{n} \sum_{l=0}^{k-1} \sigma_{l+1} \Delta_{+} h_{f}\left(u_{j}^{n+\eta_{l}}, u_{j-1}^{n+\eta_{l}}\right), \quad j \notin \bigodot^{n},
\end{array}\right.
$$

$\lambda_{j}^{n}=\Delta t^{n} / \Delta x_{j}$. The values $u_{j}^{n+1}$ are then obtained via the corrector

$$
u_{j}^{n+1}=u_{j}^{n}-\lambda_{j}^{n} \sum_{l=0}^{M-1} \sigma_{l+1} \Delta_{+} h_{f}\left(u_{j}^{n+\eta_{l}}, u_{j-1}^{n+\eta_{l}}\right)
$$

Throughout this section, we assume that the numerical flux function, $h_{f}\left(u_{1}, u_{2}\right)$, is monotone, (that is, $h_{f}$ is nonincreasing in $u_{1}$ and nondecreasing in $u_{2}$ ), locally Lipschitz continuous in both $u_{1}$ and $u_{2}$ and consistent (that is, $h_{f}(u, u)=f(u)$ ). 
We now have the following theorem:

THEOREM 3. Let $u^{\Delta}(x, t)$ be defined by (4.2), (4.3) and (4.4). Further assume that a local CFL type of restriction is satisfied. That is,

$$
\Lambda_{j}^{n+\eta_{k}}\left[\frac{h_{f}\left(u, v_{1}\right)-h_{f}\left(u, v_{2}\right)}{v_{1}-v_{2}}-\frac{h_{f}\left(v_{1}, w\right)-h_{f}\left(v_{w}, w\right)}{v_{1}-v_{2}}\right] \leqslant 1,
$$

for all $u, w, v_{1}, v_{2}$ between the values of $u_{j+1}^{n+\eta_{k}}, u_{j}^{n+\eta_{k}}, u_{j-1}^{n+\eta_{k}}$ and $\Lambda_{j}^{n+\eta_{k}}$ is defined by

$$
\Lambda_{j}^{n+\eta_{k}}= \begin{cases}\frac{\Delta t^{n}}{\Delta x_{j}}, & \text { if } j \text { or } j \pm 1 \in \mathcal{C}^{n}, \\ \sigma_{k+1} \frac{\Delta t^{n}}{\Delta x_{j}}, & \text { otherwise. }\end{cases}
$$

Then, the $\lim _{\Delta \downarrow 0} u^{\Delta}(x, t)$ exists in $L_{\mathrm{loc}}^{1}(\mathbf{R})$ on any bounded strip $[0, T]$, and this limit is the entropy satisfying solution of (4.1).

Remark 2. For the numerical flux functions A, B and C (see Eqs. (3.14), (3.16) and (3.17), resp.), the restriction (4.5) above reduces to

$$
\Lambda_{j}^{n+\eta_{k}}\left|f^{\prime}(u)\right| \leqslant 1
$$

for all $u$ between the values of $u_{j+1}^{n+\eta_{k}}, u_{j}^{n+\eta_{k}}, u_{j-1}^{n+\eta_{k}}$.

Proof of Theorem 3. The first step is to establish the following inequalities:

$$
\begin{gathered}
\left\|u^{\Delta}\left(\cdot, t^{n}\right)\right\|_{L^{\infty}(\mathbf{R})} \leqslant C_{1}, \\
\left\|u^{\Delta}\left(\cdot, t^{n}\right)\right\|_{L^{\prime}(\mathbf{R})} \leqslant C_{2}, \\
\operatorname{Var}\left(u^{\Delta}\left(\cdot, t^{n}\right)\right) \leqslant C_{3}, \\
\left\|u^{\Delta}\left(\cdot, t^{n}+\tau\right)-u^{\Delta}\left(\cdot, t^{n}\right)\right\|_{L^{\prime}(\mathbf{R})} \leqslant C_{4}(\tau+\Delta),
\end{gathered}
$$

where $C_{1}, C_{2}, C_{3}, C_{4}$ are constants independent of $\Delta>0$ and $t^{n} \leqslant T$. At $t=0$, (4.8.1) through (4.8.3) are valid for all $\Delta>0$ if $w_{0}(x) \in L^{\infty} \cap L^{1} \cap B V$ and if $u^{\Delta}(x, 0)$ is defined by averaging. That is,

$$
u^{\Delta}(x, 0)=\frac{1}{\Delta x_{j}} \int_{x_{j-1 / 2}}^{x_{j+1 / 2}} w_{0}(s) d s,
$$

when $x \in\left[x_{j-1 / 2}, x_{j+1 / 2}\right)$; see [15].

We shall prove (4.8.1) and (4.8.2) together. For $j \in \mathcal{C}^{n}$, we use (4.3), (4.4) to obtain

$$
\begin{aligned}
u_{j}^{n+1} & =\sum_{l=0}^{M-1} \sigma_{l+1}\left[u_{j}^{n+\eta_{l}}-\lambda_{j}^{n} \Delta_{+} h_{f}\left(u_{j}^{n+\eta_{l}}, u_{j-1}^{n+\eta_{l}}\right)\right] \\
& \equiv \sum_{l=0}^{M-1} \sigma_{l+1} G\left(u_{j+1}^{n+\eta_{l}}, u_{j}^{n+\eta_{l}}, u_{j-1}^{n+\eta_{l}} ; \lambda_{j}^{n}\right),
\end{aligned}
$$

where the definition of $G$ is implied. It should be noted that in the case above we have used the fact that $u_{j}^{n+\eta_{l}}=u_{j}^{n}$ for $0 \leqslant l \leqslant M-1$. For $j \notin \bigodot^{n}$ we find that

$$
\begin{aligned}
u_{j}^{n+1} & =u_{j}^{n+\eta_{M-1}}-\sigma_{M} \lambda_{j}^{n} \Delta_{+} h_{f}\left(u_{j}^{n+\eta_{M-1}}, u_{j-1}^{n+\eta_{M-1}}\right) \\
& \equiv G\left(u_{j+1}^{n+\eta_{M-1}}, u_{j}^{n+\eta_{M-1}}, u_{j-1}^{n+\eta_{M-1}} ; \sigma_{M} \lambda_{j}^{n}\right) .
\end{aligned}
$$


It can easily be verified that (4.5), along with the use of monotone flux functions, implies that $G$ is a monotone operator. More precisely, $G(\cdot, \cdot, \cdot ; \nu)$ is a nondecreasing function of its first three arguments. However, because our CFL restriction is local, we can only guarantee that each $G(\cdot, \cdot, \cdot ; \nu)$ in (4.9) and (4.10) is monotone in the range of its first three arguments.

Inequality (4.8.1) follows directly from a maximum principle implied by $G$. Let

$$
a_{j}^{n+\eta_{\iota}}=\min \left(u_{j+1}^{n+\eta_{\iota}}, u_{j}^{n+\eta_{\iota}}, u_{j-1}^{n+\eta_{l}}\right) \quad \text { and } \quad b_{j}^{n+\eta_{\iota}}=\max \left(u_{j+1}^{n+\eta_{l}}, u_{j}^{n+\eta_{\iota}}, u_{j-1}^{n+\eta_{l}}\right) \text {. }
$$

We then have the following maximum (minimum) principle:

$$
\begin{aligned}
& a_{j}^{n+\eta_{\iota}}=G\left(a_{j}^{n+\eta_{\iota}}, a_{j}^{n+\eta_{\iota}}, a_{j}^{n+\eta_{\iota}} ; \nu_{j}^{n+\eta_{\iota}}\right) \\
& \leqslant G\left(u_{j+1}^{n+\eta_{l}}, u_{j}^{n+\eta_{l}}, u_{j-1}^{n+\eta_{l}} ; \nu_{j}^{n+\eta_{l}}\right) \\
& \leqslant G\left(b_{j}^{n+\eta_{\iota}}, b_{j}^{n+\eta_{\iota}}, b_{j}^{n+\eta_{\iota}} ; \nu_{j}^{n+\eta_{\iota}}\right)=b_{j}^{n+\eta_{\iota}} \text {, }
\end{aligned}
$$

where

$$
\nu_{j}^{n+\eta_{l}}= \begin{cases}\lambda_{j}^{n}, & \text { if } j \in \mathbb{C}^{n}, \\ \sigma_{l+1} \lambda_{j}^{n}, & \text { if } j \notin \mathbb{C}^{n} .\end{cases}
$$

Inequality (4.11) together with a simple calculation easily establishes (4.8.1).

To establish inequality (4.8.2) we shall derive the pointwise inequality:

$$
\left|u_{j}^{n+1}-c\right| \leqslant\left|u_{j}^{n}-c\right|-\lambda_{j}^{n} \sum_{l=0}^{M-1} \sigma_{l+1} \Delta_{+}\left(\left|A_{j}^{n+\eta_{l}}\right|-\left|B_{j}^{n+\eta_{l}}\right|\right),
$$

where

$A_{j}=h_{f}\left(u_{j}, u_{j-1}\right)-h_{f}\left(u_{j}, c\right), \quad B_{j}=h_{f}\left(u_{j}, c\right)-h_{f}(c, c), c=$ any real number.

See [2] for the analogous uniform grid result.

Three preliminary facts will prove useful.

Fact I. Given (4.5), where $\Lambda_{j}^{n+\eta_{k}}$ is replaced by $\max (\alpha, \beta)$, we have

$$
\begin{aligned}
& \left|\left(v_{1}-v_{2}\right)-\alpha\left(h_{f}\left(u, v_{1}\right)-h_{f}\left(u, v_{2}\right)\right)+\beta\left(h_{f}\left(v_{1}, w\right)-h_{f}\left(v_{2}, w\right)\right)\right| \\
& \quad=\left|v_{1}-v_{2}\right|-\alpha\left|h_{f}\left(u, v_{1}\right)-h_{f}\left(u, v_{2}\right)\right|-\beta\left|h_{f}\left(v_{1}, w\right)-h_{f}\left(v_{2}, w\right)\right| .
\end{aligned}
$$

The proof of this fact is obvious in view of (4.5) and the monotonicity of $h_{f}$.

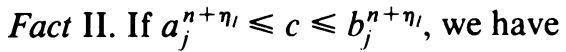

$$
\begin{aligned}
& \left|G\left(u_{j+1}^{n+\eta_{\iota}}, u_{j}^{n+\eta_{\iota}}, u_{j-1}^{n+\eta_{\iota}} ; \nu_{j}^{n+\eta_{\iota}}\right)-c\right| \\
& \quad \leqslant\left|u_{j}^{n+\eta_{\iota}}-c\right|-\nu_{j}^{n+\eta_{\iota}} \Delta_{+}\left(\left|A_{j}^{n+\eta_{\iota}}\right|-\left|B_{j}^{n+\eta_{\iota}}\right|\right) .
\end{aligned}
$$

To prove II we note the definition of $G$ to write the left-hand side of (4.14) as

$$
\left|\left(u_{j}^{n+\eta_{\iota}}-c\right)-\nu_{j}^{n+\eta_{\iota}} \Delta_{+}\left(A_{j}^{n+\eta_{\iota}}+B_{j}^{n+\eta_{\iota}}\right)\right| .
$$

Using the triangle inequality, this can be bounded above by

$$
\left|\left(u_{j}^{n+\eta_{l}}-c\right)-\nu_{j}^{n+\eta_{l}} A_{j+1}^{n+\eta_{l}}+\nu_{j}^{n+\eta_{I}} B_{j}^{n+\eta_{l}}\right|+\nu_{j}^{n+\eta_{l}}\left|A_{j}^{n+\eta_{l}}\right|+\nu_{j}^{n+\eta_{l}}\left|B_{j+1}^{n+\eta_{l}}\right| .
$$

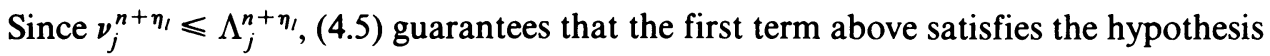
of Fact I. Therefore, applying Fact I completes the proof of Fact II. 
Fact III. The conclusion of Fact III remains valid for $\forall c \in \mathbf{R}$. The proof follows by observing that, in view of the maximum (minimum) principle (4.11), inequality (4.14) becomes an equality for $c \notin\left[a_{j}^{n+\eta_{l}}, b_{j}^{n+\eta_{l}}\right]$.

Fact III applied to (4.9) yields inequality (4.12) for the case $j \in e^{n}$. Fact III applied to (4.10) recursively yields (4.12) for the case when $j \notin \bigodot^{n}$. Now, if we set $c=0$ in (4.12), multiply this by $\Delta x_{j}$ and sum the result on $j$, we find that the $L^{1}$ norm $u^{\Delta}\left(x, t^{n}\right)$ is nonincreasing. This establishes estimate (4.8.2).

We next prove the key estimate of this paper. We shall establish estimate (4.8.3). Define

$$
C_{j}=\lambda_{j}\left(h_{f}\left(u_{j}, u_{j}\right)-h_{f}\left(u_{j}, u_{j-1}\right)\right)
$$

and

$$
D_{j}=\lambda_{j}\left(h_{f}\left(u_{j+1}, u_{j}\right)-h_{f}\left(u_{j}, u_{j}\right)\right)
$$

In the case when $j$ and $j+1$ belong to $e^{n}$, we use (4.3), (4.4) to obtain the identity

$$
u_{j+1}^{n+1}-u_{j}^{n+1}=u_{j+1}^{n}-u_{j}^{n}-\sum_{l=0}^{M-1} \sigma_{l+1} \Delta_{+}\left(C_{j}^{n+\eta_{l}}+D_{j}^{n+\eta_{l}}\right),
$$

which, as in (4.9), can be written as

$$
u_{j+1}^{n+1}-u_{j}^{n+1}=\sum_{l=0}^{M-1} \sigma_{l+1}\left[\left(u_{j+1}^{n+\eta_{l}}-u_{j}^{n+\eta_{l}}\right)-\Delta_{+}\left(C_{j}^{n+\eta_{l}}+D_{j}^{n+\eta_{l}}\right)\right] .
$$

One further preliminary fact is necessary:

Fact IV. Suppose $0 \leqslant \alpha \leqslant 1,0 \leqslant \beta \leqslant 1$ and are chosen sufficiently small such that Fact I may be applied. We then have

$$
\begin{aligned}
& \left|u_{j+1}-u_{j}-\alpha\left(C_{j+1}+D_{j+1}\right)+\beta\left(C_{j}+D_{j}\right)\right| \\
& \quad \leqslant\left|u_{j+1}-u_{j}\right|-\alpha\left(\left|C_{j+1}\right|-\left|D_{j+1}\right|\right)+\beta\left(\left|C_{j}\right|-\left|D_{j}\right|\right) .
\end{aligned}
$$

This follows by first noting that the left-hand side of (4.16) is bounded above by

$$
\left|u_{j+1}-u_{j}-\alpha C_{j+1}+\beta D_{j}\right|+\beta\left|C_{j}\right|+\alpha\left|D_{j+1}\right| \text {. }
$$

Applying the result of Fact I to this first term above completes the proof of Fact IV.

We now apply Fact IV to Eq. (4.15). Taking the absolute value of both sides of (4.15) gives us that

$$
\left|u_{j+1}^{n+1}-u_{j}^{n+1}\right| \leqslant \sum_{l=0}^{M-1} \sigma_{l+1}\left|\left(u_{j+1}^{n+\eta_{l}}-u_{j}^{n+\eta_{l}}\right)-\Delta_{+}\left(C_{j}^{n+\eta_{l}}+D_{j}^{n+\eta_{l}}\right)\right| .
$$

The CFL restriction (4.5) is assumed to be satisfied above. Therefore, we may apply Fact IV, with $\alpha=\beta=1$, to obtain

$$
\begin{aligned}
\left|u_{j+1}^{n+1}-u_{j}^{n+1}\right| & \leqslant \sum_{l=0}^{M-1} \sigma_{l+1}\left[\left|u_{j+1}^{n+\eta_{l}}-u_{j}^{n+\eta_{l}}\right|-\Delta_{+}\left(\left|C_{j}^{n+\eta_{l}}\right|-\left|D_{j}^{n+\eta_{l}}\right|\right)\right] \\
& =\left|u_{j+1}^{n}-u_{j}^{n}\right|-\sum_{l=0}^{M-1} \sigma_{l+1} \Delta_{+}\left(\left|C_{j}^{n+\eta_{l}}\right|-\left|D_{j}^{n+\eta_{l}}\right|\right) .
\end{aligned}
$$


For $j$ and $j+1$ not contained in $e^{n}$, we may write the identity

$$
u_{j+1}^{n+1}-u_{j}^{n+1}=u_{j+1}^{n+\eta_{M-1}}-u_{j}^{n+\eta_{M-1}}-\sigma_{M} \Delta_{+}\left(C_{j}^{n+\eta_{M-1}}+D_{j}^{n+\eta_{M-1}}\right) .
$$

For this range of $j$ we may apply Fact IV with $\alpha=\beta=\sigma_{M}$, yielding the inequality

$$
\left|u_{j+1}^{n+1}-u_{j}^{n+1}\right| \leqslant\left|u_{j+1}^{n+\eta_{M-1}}-u_{j}^{n+\eta_{M-1}}\right|-\sigma_{M} \Delta_{+}\left(\left|C_{j}^{n+\eta_{M-1}}\right|-\left|D_{j}^{n+\eta_{M-1}}\right|\right) .
$$

Repeating this argument inductively leads us again to (4.18).

The nontrivial cases occur at the interface; that is for $j \in \mathcal{C}^{n}$ and $j+1 \notin \bigodot^{n}$ or for $j \notin \mathcal{C}^{n}$ and $j+1 \in \mathcal{C}^{n}$. We consider only the former case since the latter follows in a symmetric fashion.

The corrector (4.4) gives us

$$
u_{j+1}^{n+1}-u_{j}^{n+1}=u_{j+1}^{n}-u_{j}^{n}-\sum_{l=0}^{M-1} \sigma_{l+1} \Delta_{+}\left(C_{j}^{n+\eta_{l}}+D_{j}^{n+\eta_{l}}\right),
$$

which can be written as

$$
\begin{gathered}
\sum_{l=0}^{M-1} \sigma_{l+1}\left[\left(u_{j+1}^{n+\eta_{l}}-u_{j}^{n+\eta_{l}}\right)-\Delta_{+}\left(C_{j}^{n+\eta_{l}}+D_{j}^{n+\eta_{l}}\right)\right] \\
+\sum_{l=0}^{M-1} \sigma_{l+1}\left[\left(u_{j+1}^{n}-u_{j}^{n}\right)-\left(u_{j+1}^{n+\eta_{l}}-u_{j}^{n+\eta_{l}}\right)\right] .
\end{gathered}
$$

Using the predictor (4.3), we have that

$$
\begin{aligned}
\sum_{l=0}^{M-1} \sigma_{l+1}\left[\left(u_{j+1}^{n}-u_{j}^{n}\right)\right. & \left.-\left(u_{j+1}^{n+\eta_{l}}-u_{j}^{n+\eta_{l}}\right)\right] \\
& =\sum_{l=0}^{M-1} \sigma_{l+1}\left[\sum_{k=0}^{l-1} \sigma_{k+1}\left(C_{j+1}^{n+\eta_{k}}+D_{j+1}^{n+\eta_{k}}\right)\right] .
\end{aligned}
$$

Reversing the order of summation, this becomes

$$
\sum_{l=0}^{M-1}\left(1-\eta_{l+1}\right) \sigma_{l+1}\left(C_{j+1}^{n+\eta_{l}}+D_{j+1}^{n+\eta_{l}}\right)
$$

Substituting (4.20) into (4.19) gives us

$$
\begin{aligned}
& u_{j+1}^{n+1}-u_{j}^{n+1} \\
& \quad=\sum_{l=0}^{M-1} \sigma_{l+1}\left[\left(u_{j+1}^{n+\eta_{l}}-u_{j}^{n+\eta_{l}}\right)-\eta_{l+1}\left(C_{j+1}^{n+\eta_{l}}+D_{j+1}^{n+\eta_{l}}\right)+\left(C_{j}^{n+\eta_{l}}+D_{j}^{n+\eta_{l}}\right)\right] .
\end{aligned}
$$

Again using Fact IV, with $\alpha=\eta_{l+1}$ and $\beta=1$, we obtain

$$
\begin{aligned}
& \left|u_{j+1}^{n+1}-u_{j}^{n+1}\right| \\
& \leqslant \sum_{l=0}^{M-1} \sigma_{l+1}\left[\left|u_{j+1}^{n+\eta_{l}}-u_{j}^{n+\eta_{l}}\right|-\eta_{l+1}\left(\left|C_{j+1}^{n+\eta_{l}}\right|-\left|D_{j+1}^{n+\eta_{l}}\right|\right)+\left(\left|C_{j}^{n+\eta_{l}}\right|-\left|D_{j}^{n+\eta_{l}}\right|\right)\right] .
\end{aligned}
$$


Furthermore, from the predictor (4.3) and Fact IV with $\alpha=\sigma_{l}$ and $\beta=0$, we have that

$$
\begin{aligned}
\left|u_{j+1}^{n+\eta_{l}}-u_{j}^{n+\eta_{l}}\right| & =\left|u_{j+1}^{n+\eta_{l-1}}-\sigma_{l}\left(C_{j+1}^{n+\eta_{l-1}}+D_{j+1}^{n+\eta_{l-1}}\right)-u_{j}^{n+\eta_{l-1}}\right| \\
& \leqslant\left|u_{j+1}^{n+\eta_{l-1}}-u_{j}^{n+\eta_{l-1}}\right|-\sigma_{l}\left(\left|C_{j+1}^{n+\eta_{l-1}}\right|-\left|D_{j+1}^{n+\eta_{l-1}}\right|\right) .
\end{aligned}
$$

Repeating the above argument inductively, we arrive at the inequality

$$
\left|u_{j+1}^{n+\eta_{1}}-u_{j}^{n+\eta_{1}}\right| \leqslant\left|u_{j+1}^{n}-u_{j}^{n}\right|-\sum_{k=0}^{1-1} \sigma_{k+1}\left(\left|C_{j+1}^{n+\eta_{k}}\right|-\left|D_{j+1}^{n+\eta_{k}}\right|\right) .
$$

Substitute (4.22) into (4.21) and exchange the order of summation on that result to obtain

$$
\left|u_{j+1}^{n+1}-u_{j}^{n+1}\right| \leqslant\left|u_{j+1}^{n}-u_{j}^{n}\right|-\sum_{l=0}^{M-1} \sigma_{l+1} \Delta_{+}\left(\left|C_{j}^{n+\eta_{l}}\right|-\left|D_{j}^{n+\eta_{l}}\right|\right) .
$$

Inequality (4.23) is now verified for all $j$. Summing this on $j$ shows that the $\operatorname{Var}\left(u^{\Delta}\left(x, t^{n}\right)\right)$ is nonincreasing, establishing (4.8.3).

Finally, we shall outline the proof of (4.8.4). From (4.3) we have the obvious inequality

$$
\left|u_{j+1}^{n+\eta_{l}}-u_{j}^{n+\eta_{1}}\right| \leqslant(1+K)^{l} \sum_{k=-l}^{l}\left|u_{j+k+1}^{n}-u_{j+k}^{n}\right| .
$$

Here, $K$ is the Lipschitz constant for $h_{f}$. (4.24) shows the variation remains bounded at all intermediate time steps, $t^{n+\eta_{\ell}}$. For any conservation form difference scheme, approximating a scalar conservation law, (4.8.4) follows directly from variation boundedness. For details see [15] or [2].

It is widely known that (4.8.1) through (4.8.4) implies that every sequence of $\left\{u^{\Delta}\right\}$, with $\Delta$ tending to zero, has a convergent subsequence in the space $L^{\infty}\left([0, T] ; L_{\text {loc }}^{1}(\mathbf{R})\right)$. See [15], or [2]. What remains to be shown is that the limit of each subsequence satisfies the entropy condition, as used by Kruzkov [9]. This entropy condition implies both the uniqueness of each subsequence's limit and that the limit is a weak, entropy satisfying, solution of (4.1).

To complete the proof of Theorem 3, we therefore need only show for all $\varphi \in C_{0}^{1}\left(\mathbf{R} \times \mathbf{R}^{+}\right), \varphi \geqslant 0$ and all real numbers $c$, that

$$
-\lim _{\Delta \downarrow 0} \int_{\mathbf{R} \times \mathbf{R}^{+}}\left|u^{\Delta}-c\right| \varphi_{t}+\operatorname{sgn}\left(u^{\Delta}-c\right)\left(f\left(u^{\Delta}\right)-f(c)\right) \varphi_{x} d x d t \leqslant 0 .
$$

Recall inequality (4.12). This can be written as

$$
\Delta_{+}^{t}\left|u_{j}^{n}-c\right|+\lambda_{j}^{n} \sum_{l=0}^{M-1} \sigma_{l+1} \Delta_{+}^{x}\left(\left|A_{j}^{n+\eta_{l}}\right|-\left|B_{j}^{n+\eta_{l}}\right|\right) \leqslant 0 .
$$

Now, observe that

$$
\begin{aligned}
\left|A_{j}\right|-\left|B_{j}\right|= & \operatorname{sgn}\left(u_{j-1}-c\right)\left(h_{f}\left(u_{j}, u_{j-1}\right)-h_{f}\left(u_{j}, c\right)\right) \\
& +\operatorname{sgn}\left(u_{j}-c\right)\left(h_{f}\left(u_{j}, c\right)-h_{f}(c, c)\right) .
\end{aligned}
$$


Next, multiply (4.26) by $\varphi\left(x_{j}, t^{n}\right) \Delta x_{j}$ and sum by parts over $j$ and $n$. Consistency implies that the right-hand side of (4.27) tends to

$$
\operatorname{sgn}(u-c)(f(u)-f(c))
$$

boundedly a.e. The remainder of the proof follows in the same fashion as the proof of the Lax-Wendroff Theorem. See [12].

\author{
Department of Mathematics \\ University of California \\ Los Angeles, California 90024 \\ Department of Mathematics \\ University of Southern California \\ Los Angeles, California 90007
}

1. S. Chakravarthy \& S. Osher, Numerical Experiments With the Osher Upwind Scheme for the Euler Equations, AIAA-82-0975, St. Louis, Mo., June 1982.

2. M. G. CRANDall \& A. Majda, "Monotone difference approximations for scalar conservation laws," Math. Comp., v. 34, 1980, pp. 1-22.

3. B. ENGQuist \& S. OSHER, "Stable and entropy satisfying approximations for transonic flow calculations," Math. Comp., v. 34, 1980, pp. 45-75.

4. B. ENGQUIST \& S. OSHER, "One sided difference approximations for nonlinear conservation laws," Math. Comp., v. 36, 1981, pp. 321-351.

5. B. Engquist, S. Osher, P. Roe \& B. Van Leer, "Discrete shocks and upwind schemes." (To appear.)

6. S. K. Godunov, "A finite-difference method for the numerical computation of discontinuous solutions of the equations of fluid dynamics," Mat. Sb., v. 47, 1959, pp. 271-290. (Russian)

7. P. M. Goorian \& R. VAN BUSKIRK, Implicit Calculations of Transonic Flows Using Monotone Methods, AIAA-81-0331, St. Louis, Mo., Jan. 1981.

8. G. Jennings, "Discrete shocks," Comm. Pure Appl. Math., v. 27, 1974, pp. 25-37.

9. S. N. KRUZKov, "First order quasi-linear equations in several independent variables," Math. USSR Sb., v. 10, 1970, pp. 217-243.

10. P. D. LAX, "Shock waves and entropy," in Contributions to Nonlinear Functional Analysis (E. H. Zarantonello, Ed.), Academic Press, New York, 1971, pp. 603-634.

11. P. D. LAX, Hyperbolic Systems of Conservation Laws and the Mathematical Theory of Shock Waves, SIAM Regional Conf. Series Lectures in Appl. Math., Vol. 11, 1972.

12. P. D. LaX \& B. Wendroff, “Systems of conservation laws," Comm. Pure Appl. Math., v. 13, 1960, pp. 217-237.

13. O. A. OleiniK, "Discontinuous solutions of non-linear differential equations," Uspekhi Mat. Nauk, v. 12, 1957, no. 3(75), pp. 3-73; English transl., Amer. Math. Soc. Transl. (2), v. 26, 1963, pp. 95- 172.

14. S. OsHer, Numerical Solution of Singular Perturbation Problems and Hyperbolic Systems of Conservation Laws, North-Holland Math. Studies \#47, 1981, pp. 179-205.

15. R. SANDERS, "On convergence of monotone finite difference schemes with variable spatial differencing," Math. Comp., v. 40, 1983, pp. 91-106.

16. B. VAN LEER, On the Relation Between the Upwind Differencing Schemes of Godunov, Engquist-Osher, and Roe, ICASE Report \#81-11, 1981. 